\title{
PRE-MOVEMENT CONTRALATERAL EEG LOW BETA POWER IS MODULATED WITH MOTOR ADAPTATION LEARNING
}

\author{
*Ozan Özdenizci, * Mustafa Yalçın, *Ahmetcan Erdoğan, *Volkan Patoğlu, \\ ${ }^{\dagger}$ Moritz Grosse-Wentrup and ${ }^{*}$ Müjdat Çetin \\ ${ }^{*}$ Faculty of Engineering and Natural Sciences, Sabanc1 University, Istanbul, Turkey \\ ${ }^{\dagger}$ Department of Empirical Inference, Max Planck Institute for Intelligent Systems, Tübingen, Germany
}

\begin{abstract}
Various neuroimaging studies aim to understand the complex nature of human motor behavior. There exists a variety of experimental approaches to study neurophysiological correlates of performance during different motor tasks. As distinct from studies based on visuomotor learning, we investigate changes in electroencephalographic (EEG) activity during an actual physical motor adaptation learning experiment. Based on statistical analysis of EEG signals collected during a force-field adaptation task performed with the dominant hand, we observe a modulation of pre-movement upper alpha $(10-12 \mathrm{~Hz})$ and lower beta $(13-16 \mathrm{~Hz})$ powers over the contralateral region. This modulation is observed to be stronger in lower beta range and, through a regression analysis, is shown to be related with motor adaptation performance on a subject-specific level.
\end{abstract}

Index Terms - EEG; motor learning; force-field adaptation; pre-movement; brain-computer interfaces

\section{INTRODUCTION}

Stroke is a common cause for restricted activities of daily living for millions of patients. People severely affected by stroke are often left in a locked-in state with sustained loss of voluntary muscle control. In order to provide valuable insights into the understanding of stroke and the neural processes in the brain related to the complex nature of human motor behavior, post-stroke recovery is often studied as a form of motor learning in several neuroimaging studies [1]. To date, various experimental approaches have been proposed to identify neurophysiological correlates of motor learning.

Several pieces of previous work have studied the concept of visuomotor learning using simultaneously recorded EEG data. Independently of the neural activity during motor execution, EEG correlates of visuomotor task performance during pre-movement phases prior to motor execution and how

This work was partially supported by the Scientific and Technological Research Council of Turkey by a graduate fellowship and under Grant 112M698, and by Sabancı University under Grant IACF-11-00889. this activity changes with visuomotor learning was particularly studied [2-7]. Importantly, visuomotor tasks require learning of an underlying mapping between the actual motor task space and the visual feedback environment [8], which further incorporates separate processing of different mapping aspects into the learning process $[9,10]$. Hence, these studies generally quantify visual mapping performance together with motor execution skill, as visuomotor learning performance. We argue that such neuroimaging studies should dissociate learning of an underlying visual mapping from the pure motor learning process. In motor rehabilitation literature, motor learning, evaluated either in the form of motor adaptation or skill learning [11], is widely studied in force-field adaptation tasks $[12,13]$. With this object in mind, we investigate how pre-movement EEG activity changes during pure motor learning without a separate artificial visual feedback environment, throughout a force-field adaptation task performed within an actual physical environment using a robotic setup.

Based on analysis of experimental data from fifteen healthy subjects, we observe a modulation of upper alpha $(10-12 \mathrm{~Hz})$ and lower beta $(13-16 \mathrm{~Hz})$ activity over the contralateral region during pre-movement phases throughout motor adaptation learning with the dominant hand. Moreover, through a statistical analysis of the recorded EEG signals and experimental data related to motor task performance, we demonstrate that this modulation is stronger in lower beta range and is associated with individual motor adaptation performances of subjects. Finally we propose to exploit these findings to potentially be used as a biomarker in novel stroke rehabilitation approaches by means of a brain-computer interface (BCI), which involves the idea of supporting motor recovery as well as inducing neural plasticity $[14,15]$.

\section{METHODS}

\subsection{Subjects and Experimental Data}

Fifteen right handed healthy subjects (10 male, 5 female; mean age $23.73 \pm 3.03$ ) participated in this study. All subjects were naive to the force-field adaptation task. Before the experiments, all participants gave their informed consent 
after the experimental procedure was explained to them in accordance with guidelines set by the research ethics committee of Sabanc1 University.

Throughout the experiments, the robotic setup recorded data at $500 \mathrm{~Hz}$ sampling rate and a 64-channel EEG was recorded at $512 \mathrm{~Hz}$ sampling rate, using active EEG electrodes and a BioSemi ActiveTwo amplifier (Biosemi Inc., Amsterdam, The Netherlands). Electrodes were placed according to the 10-20 system. All data were re-referenced to common average reference offline.

\subsection{Study Design}

Participating subjects performed a force-field adaptation task under simultaneous EEG recordings with the goal of performing planar center-out reaching movements under an unknown force-field, as straightly as possible. During the experiments, subjects sat in front of a horizontally placed board, while holding an end-effector with their right hands that was suspended from above onto the board (see Figure 1). The end-effector was attached to a 3 degrees-of-freedom modified delta robot with constrained motion on $\mathrm{z}$-axis and was only capable of two-dimensional movements that were restricted to fall within a circle with a radius of $200 \mathrm{~mm}$. Idle starting position of the end-effector corresponded to the center of this circle. The four target locations placed on the circle at the northeast, northwest, southeast, and southwest positions were indicated with holes over the board containing LEDs inside.

Before the experiments, all subjects performed a pre-flight phase of eight trials (i.e., reaching movements) without any force-field to get familiar with the task workspace and trial flow. As part of the force-field adaptation task, each subject performed 200 trials in total, which were divided into three blocks of 40, 80, and 80 trials. Within each of these blocks, there were equal number of trials per target location. After the task, subjects also performed a washout phase of 20 trials which involved no force-field. Additionally, throughout the experiment, four blocks of five minute resting-state EEG recordings were performed; first resting-state recording before the force-field adaptation task, second recording after the

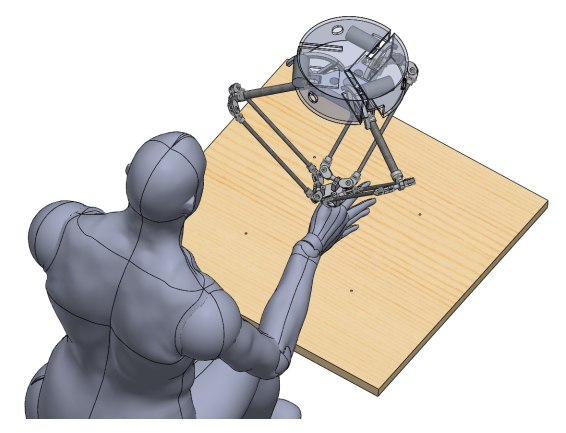

Fig. 1. Illustration of the task workspace. Four target locations are placed on the board, each at $200 \mathrm{~mm}$ distance. first block of 40 trials, third recording at the end of the forcefield adaptation task, and fourth resting-state recording after the washout phase. During these recordings, subjects were placed approximately 1.5 meters in front of a computer screen and instructed to relax with eyes open. Same experimental setup and data were also presented and used in our previous work for a different analysis [16].

\subsection{Force-Field Adaptation Task}

During reaching movements within the task workspace, a velocity dependent force-field was applied to the end-effector by the robotic setup. Specifically, end-effector velocity vector $\vec{v}$ was multiplied with a constant matrix $\mathbf{B}$, representing the viscosity of the imposed environment, to compute $\vec{f}=\mathbf{B} \vec{v}$ at each time point, where $\vec{f}$ represented the forces that the robotic setup is programmed to produce on the end-effector as the subject performed reaching movements. The constant matrix $\mathbf{B}$ was the same as in [17].

Each trial began with a planning phase, where the subjects were instructed to hold the end-effector at the starting position and plan the upcoming movement. The planning phase lasted 2.5-3.5 seconds, chosen randomly from a uniform distribution. Within the first second of this phase, the robotic setup assisted the subjects to center the end-effector position and directed the end-effector to the pre-calibrated starting position. During the planning phase, one of the four possible targets was selected by the system randomly and indicated by a blinking LED light. Each trial began with a new target location. At the end of the planning phase, the LED turned on steadily, signaling the beginning of the go phase. The time interval after the first second, until the end of the planning phase is referred as the pre-movement phase.

In the go phase, subjects were instructed to reach for the target by moving the end-effector over the board. A trial was considered complete when the subject moved the end-effector to within $20 \mathrm{~mm}$ of the target or if the subject exceeded a time limit of 3 seconds. After the go phase, the subjects were instructed to move the end-effector back to the starting position. At the end of the trials, to quantify motor adaptation amount, a calculated auditory feedback score within a range of 0-100 was provided from a speaker. The score in each trial indicated how straight the movement trajectory was in the corresponding trial. The area between the curve defined by the movement trajectory and a straight line to the target served as the basis kinematic measure for the score [18]. Aim of the subjects was to increase the feedback score throughout trials.

\subsection{EEG Artifact Correction}

In order to identify and attenuate potential artifactual activity from the EEG as part of pre-processing, we employed independent component analysis (ICA) [19]. We pooled all resting-state EEG data from all subjects, by concatenating 
high pass filtered data at $3 \mathrm{~Hz}$, and separated this data into group-wise statistically independent components (ICs) that represent cortical patterns consistently found across all subjects. This was done by first transforming the data into 64 principal components and then running the SOBI-algorithm, which computes the ICs [20]. We inspected each IC's topography, spectrum and time-series manually and rejected those which were not of cortical origin [21]. We then reprojected the remaining ICs to the scalp.

\subsection{Pre-Movement EEG Activity Modulation}

Using the artifact-corrected EEG data, each subject's premovement activity (i.e., EEG signals extracted from the planning phase of each trial) was transformed into the spectral domain. Specifically, we computed spectral powers of data from each electrode during pre-movement as mean log-bandpowers in sixteen frequency sub-bands. Log-bandpowers were computed at center frequencies from $9 \mathrm{~Hz}$ to $24 \mathrm{~Hz}$, in $3 \mathrm{~Hz}$-wide bands, using an FFT in conjunction with a Hanning window of one second length and a step-size of $100 \mathrm{~ms}$.

To observe any frequency sub-band specific modulation in data from any particular region of the head surface throughout the experiments, we computed mean log-bandpowers across all subjects, at each electrode and frequency sub-band during each trial. For each frequency sub-band, at each electrode, $R^{2}$ between mean log-bandpowers at each trial and temporal order of 200 trials was computed. A higher $R^{2}$ value denoted a stronger modulation of activity at that particular electrode. Then, for each frequency sub-band, $R^{2}$ values corresponding to each electrode was displayed as a modulation topography.

\subsection{Relation of Modulation with Motor Adaptation}

Frequency sub-bands and electrodes that showed modulation on the topographies were further inspected on whether this pre-movement modulation is associated with motor adaptation learning on a subject-specific level. For this purpose, firstly motor adaptation performance was quantified with four different kinematic measures in every trial of all subjects: (1) auditory feedback scores, (2) total area between the curve defined by the movement trajectory and a straight line to the target, (3) maximum deflection from the straight line path to the target during movement, (4) coefficient of determination between vertical and horizontal position vectors during reaching movement.

Then, all electrodes and frequency sub-bands that showed modulation were investigated individually for each subject on whether any of those activities is correlated with motor adaptation across trials. Specifically, the four kinematic measures computed at each trial served as the independent variables to a multivariate linear regression model and measured electrode sub-band powers at each trial served as the dependent variables, to predict EEG powers for 200 trials. For every subject, the electrode and frequency sub-band pair that showed maximum $R^{2}$ between measured electrode sub-band powers and the corresponding model's predicted EEG powers across 200 trials was determined. Significance of this $R^{2}$ was tested with a random permutation test. To test the null-hypothesis of zero correlation, we randomly permuted the assignment of kinematic measures to EEG powers across trials 10,000 times and estimated the frequency at which the prediction model achieved a higher $R^{2}$ than with the true assignment of EEG powers to kinematic measures as the $p$-value.

\section{RESULTS}

Modulation topographies across frequency sub-bands with center frequencies from $9 \mathrm{~Hz}$ to $24 \mathrm{~Hz}$ showed apparent modulation in upper alpha $(10-12 \mathrm{~Hz})$ and lower beta $(13-16 \mathrm{~Hz})$ frequencies over the contralateral region (see Figure 2). This modulation was stronger and significant in lower beta range; highest for electrode $\mathrm{C} 1$ at sub-band center frequency $15 \mathrm{~Hz}$ ( $R^{2}=0.16, p<10^{-3}$ for a random permutation test). Here, the correlation coefficient between mean log-bandpowers and temporal order of trials was positive $(\rho=0.40)$, indicating an increase of activity rather than a suppression.
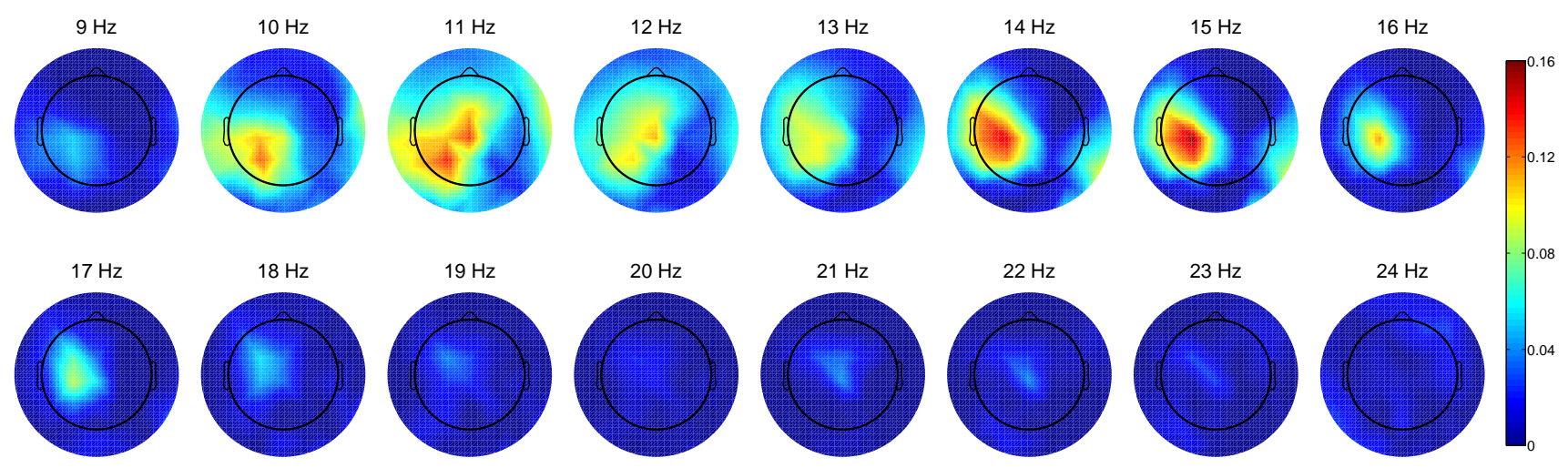

Fig. 2. Modulation topographies of bandpowers in $3 \mathrm{~Hz}$-wide sub-bands with center frequencies ranging from $9 \mathrm{~Hz}$ to $24 \mathrm{~Hz}$. 
Table 1. Subject-level most significant results of $R^{2}$ values between motor adaptation performance and modulation in low beta sub-bands over the contralateral region.

\begin{tabular}{cccccccccccccccc}
\hline Subjects & S1 & S2 & S3 & S4 & S5 & S6 & S7 & S8 & S9 & S10 & S11 & S12 & S13 & S14 & S15 \\
\hline Electrode Location & CP5 & C3 & C1 & FC1 & CP3 & CP5 & CP1 & FC1 & CP5 & FC5 & CP1 & C1 & CP1 & CP1 & FC1 \\
Center Frequency $(\mathbf{H z})$ & 15 & 13 & 16 & 14 & 15 & 15 & 14 & 13 & 14 & 15 & 15 & 16 & 13 & 13 & 13 \\
R-Squared $\left(R^{2}\right)$ & $\mathbf{0 . 0 5}^{*}$ & $\mathbf{0 . 0 5}$ & 0.03 & 0.04 & $\mathbf{0 . 0 5}^{*}$ & $\mathbf{0 . 0 7}^{\dagger}$ & $\mathbf{0 . 0 6}^{*}$ & $\mathbf{0 . 0 6}^{*}$ & $\mathbf{0 . 0 5}^{*}$ & 0.04 & 0.04 & $\mathbf{0 . 0 6}^{*}$ & $\mathbf{0 . 1 7}^{\dagger}$ & 0.03 & $\mathbf{0 . 0 7}^{\dagger}$ \\
\hline
\end{tabular}

${ }^{*} p<0.05,{ }^{\dagger} p<0.01$

Based on this initial analysis, six electrodes over the contralateral region $(\mathrm{C} 1, \mathrm{C} 3, \mathrm{C} 5, \mathrm{CP} 1, \mathrm{CP} 3, \mathrm{CP} 5, \mathrm{FP} 1, \mathrm{FP} 3, \mathrm{FP} 5$ sites from the 10-20 electrode placement system [22]) in four frequency sub-bands in low beta range (with center frequencies from $13 \mathrm{~Hz}$ to $16 \mathrm{~Hz}$ ) were selected for subject-specific analyses on the relation of EEG activity modulation and motor adaptation learning. For each subject, the electrode and frequency sub-band pair with highest $R^{2}$ values among these are presented in Table 1 with the corresponding $p$-values. Ten out of fifteen subjects showed specific pre-movement low beta activity over the contralateral region that is significantly correlated with trial-wise motor adaptation performance. Moreover, we observe that a whole low beta band $(13-16 \mathrm{~Hz})$ modulation topography of the mean activity over these ten subjects showed stronger modulation than the other five subjects' mean activity modulation topography (see Figure 3 ).

\section{DISCUSSION}

In this study we implemented a force-field adaptation task with simultaneous EEG recordings to study the changes in neural activity during motor adaptation learning. The task was performed within an actual physical environment as distinct from conventional neuroimaging studies based on visuomotor learning tasks. Using an ICA-based artifact removal procedure and an EEG signal processing pipeline, we observed a modulation of contralateral upper alpha and lower

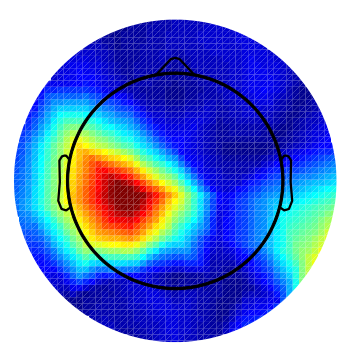

(a)

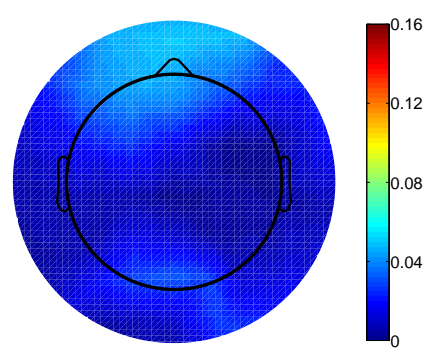

(b)
Fig. 3. Low beta modulation topographies for mean activity of the: (a) ten subjects with significant correlation between modulation and motor performance, (b) other five subjects. beta powers throughout motor adaptation learning, which was strongly evident for lower beta range. Moreover using a multivariate linear regression approach, we present that this modulation is associated with motor learning on a subject-specific level. This demonstrates that it might be possible to predict motor learning performance from EEG data.

We argue that these findings can be used as a biomarker for current BCI-assisted stroke rehabilitation approaches. In such protocols, BCIs are often used to decode movement intent from EEG data that is synchronized to a rehabilitation robot with haptic feedback to provide movement support during rehabilitation exercises [23-25]. Similarly during rehabilitation exercises a BCI can monitor the EEG of the patient, and provide movement support whenever an individually spatially and spectrally characterized increase of pre-movement EEG activity is detected, with the goal of supporting motor learning. This artificial neurofeedback loop can possibly further incorporate adaptive approaches as proposed in [26], or likewise studied in [27, 28]. However, whether reinforcing this change in pre-movement activity by such rewards would indeed support motor recovery of stroke patients remains as an interesting question inspired by the study presented here.

\section{REFERENCES}

[1] J. W. Krakauer, "Motor learning: its relevance to stroke recovery and neurorehabilitation," Current Opinion in Neurology, vol. 19, no. 1, pp. 84-90, 2006.

[2] T. Meyer, J. Peters, T. O. Zander, B. Schölkopf, and M. Grosse-Wentrup, "Predicting motor learning performance from electroencephalographic data," Journal of NeuroEngineering and Rehabilitation, vol. 11, no. 1, 2014.

[3] L. Pitto et al., "Neural correlates of motor learning and performance in a virtual ball putting task," in IEEE ICORR, 2011, pp. 1-6.

[4] L. Yang et al., "Alpha and beta band power changes predict reaction time and endpoint error during planning reaching movements," in 7th IEEE International Conference on Biomedical Engineering and Informatics, 2014, pp. 264-268. 
[5] A. Meinel, S. Castaño-Candamil, J. Reis, and M. Tangermann, "Pre-trial EEG-based single-trial motor performance prediction to enhance neuroergonomics for a hand force task," Frontiers in Human Neuroscience, vol. 10, 2016.

[6] B. Pollok et al., "Changes of motor-cortical oscillations associated with motor learning," Neuroscience, vol. 275, pp. 47-53, 2014.

[7] A. A. Kühn et al., "Event-related beta desynchronization in human subthalamic nucleus correlates with motor performance," Brain, vol. 127, no. 4, pp. 735-746, 2004.

[8] U. Sailer, J. R. Flanagan, and R. S. Johansson, "Eyehand coordination during learning of a novel visuomotor task," The Journal of Neuroscience, vol. 25, no. 39, pp. 8833-8842, 2005.

[9] J. W. Krakauer, Z. M. Pine, M.-F. Ghilardi, and C. Ghez, "Learning of visuomotor transformations for vectorial planning of reaching trajectories," The Journal of Neuroscience, vol. 20, no. 23, pp. 8916-8924, 2000.

[10] R. A. Scheidt et al., "Interaction of visual and proprioceptive feedback during adaptation of human reaching movements," Journal of Neurophysiology, vol. 93, no. 6, pp. 3200-3213, 2005.

[11] J. W. Krakauer and P. Mazzoni, "Human sensorimotor learning: adaptation, skill, and beyond," Current Opinion in Neurobiology, vol. 21, no. 4, pp. 636-644, 2011.

[12] J. L. Patton and F. A. Mussa-Ivaldi, "Robot-assisted adaptive training: custom force fields for teaching movement patterns," IEEE Transactions on Biomedical Engineering, vol. 51, no. 4, pp. 636-646, 2004.

[13] F. Gandolfo, F. A. Mussa-Ivaldi, and E. Bizzi, "Motor learning by field approximation," PNAS, vol. 93, no. 9, pp. 3843-3846, 1996.

[14] J. J. Daly and J. R. Wolpaw, "Brain-computer interfaces in neurological rehabilitation," The Lancet Neurology, vol. 7, no. 11, pp. 1032-1043, 2008.

[15] M. Grosse-Wentrup, D. Mattia, and K. Oweiss, "Using brain-computer interfaces to induce neural plasticity and restore function," Journal of Neural Engineering, vol. 8, no. 2, pp. 025004, 2011.

[16] O. Özdenizci, M. Yalçın, A. Erdoğan, V. Patoğlu, M. Grosse-Wentrup, and M. Çetin, "Resting-state EEG correlates of motor learning performance in a force-field adaptation task," in IEEE SIU, 2016, pp. 2253-2256.
[17] R. Shadmehr and F. A. Mussa-Ivaldi, "Adaptive representation of dynamics during learning of a motor task," The Journal of Neuroscience, vol. 14, no. 5, pp. 32083224, 1994.

[18] N. Malfait, D. M. Shiller, and D. J. Ostry, "Transfer of motor learning across arm configurations," The Journal of Neuroscience, vol. 22, no. 22, pp. 9656-9660, 2002.

[19] T.-P. Jung et al., "Removing electroencephalographic artifacts by blind source separation," Psychophysiology, vol. 37, no. 02, pp. 163-178, 2000.

[20] A. Belouchrani, K. Abed-Meraim, J.-F. Cardoso, and E. Moulines, "A blind source separation technique using second-order statistics," IEEE Transactions on Signal Processing, vol. 45, no. 2, pp. 434-444, 1997.

[21] A. Delorme, J. Palmer, J. Onton, R. Oostenveld, and S. Makeig, "Independent EEG sources are dipolar," PloS one, vol. 7, no. 2, pp. e30135, 2012.

[22] G. H. Klem et al., "The ten-twenty electrode system of the international federation," Electroencephalogr Clin Neurophysiol, vol. 52, no. 3, 1999.

[23] M. Gomez-Rodriguez, J. Peters, J. Hill, B. Schölkopf, A. Gharabaghi, and M. Grosse-Wentrup, "Closing the sensorimotor loop: haptic feedback facilitates decoding of motor imagery," Journal of Neural Engineering, vol. 8, no. 3, pp. 036005, 2011.

[24] M. Saraç, E. Koyaş, A. Erdoğan, M. Çetin, and V. Patoğlu, "Brain computer interface based robotic rehabilitation with online modification of task speed," in IEEE ICORR, 2013, pp. 1-7.

[25] A. Ramos-Murguialday et al., "Brain-machine interface in chronic stroke rehabilitation: A controlled study," Annals of Neurology, vol. 74, no. 1, pp. 100-108, 2013.

[26] O. Özdenizci, T. Meyer, M. Çetin, and M. GrosseWentrup, "Towards neurofeedback training of associative brain areas for stroke rehabilitation," in Proceedings of the 6th International Brain-Computer Interface Conference, 2014.

[27] G. Naros and A. Gharabaghi, "Reinforcement learning of self-regulated $\beta$-oscillations for motor restoration in chronic stroke," Frontiers in Human Neuroscience, vol. 9, 2015.

[28] G. Naros, I. Naros, F. Grimm, U. Ziemann, and A. Gharabaghi, "Reinforcement learning of selfregulated sensorimotor $\beta$-oscillations improves motor performance," NeuroImage, vol. 134, pp. 142-152, 2016. 